\title{
Absolute Nucleic Acid Quantification System and Analysis
}

\author{
B.F. SONG, Q. SONG, W.JIN, Q.C.TIAN, Y. MU* \\ State Key Laboratory of Industrial Control Technology, Zhejiang University, Hangzhou, China
}

\begin{abstract}
Digital nucleic acid amplification and determination has become an attractive technique by its capable of high sensitivity and precision. With the development of microfluidic technique, this method becomes much easier to perform, with less sample requirement and cross contamination. The dynamic range of measurement is critical for digital nucleic acid amplification, and has greatly increased by improving the number of reactors. Here, a microfluidic device for digital nucleic acid amplification has been demonstrated, and the measurement uncertainty has been discussed. The paper's aim is to present an absolute nucleic acid quantification system which is fabricated by nano-fabrication, and the dynamic range as well as measurement uncertainty of the system is also presented in the paper.
\end{abstract}

KEYWORDS: Digital nucleic acid amplification; Microfluidic; Dynamic range; Measurement Uncertainty

\section{INTRODUCATION}

Polymerase Chain Reaction (PCR) is a technology in molecular biology used to amplify a piece of particular DNA sequence, and generating thousands to millions of copies.(1) This technique is developed by Kary Mullis in 1983, and now is indispensible in the field of medical and biological research.(2) The most popular PCR technique to measure the presence and concentration of a DNA sequence is real-time quantitative PCR (qPCR).(3) In qPCR, the original number of particular DNA molecules is obtained by calculating the number of amplification cycles. However, qPCR does not have the capability to distinguish copy number variants or gene expression differences smaller than about twofold.

By partitioning the diluted sample into hundreds or even millions of small-volume reaction chambers so that one or zero copies of the sequence of interest can be confined in each isolated compartment and amplified. Digital nucleic acid amplification technology transfers the traditional exponential format with errors from amplification bias or noise into an easy 'yes or no' counting, which performance is more robust.(4) The concept was first described in 1992, and named by Bert Vogelstein and Ken Kinzler in 1999.(5) This method is capable of higher sensitivity and precision, has become a powerful technique for absolute nucleic acid quantification without needing to reference any other material.(6)

Although this theory is simple, it's not easy to implementation. The initial demonstration of dPCR is performed in commercially available 384-well plates. With the help of mcirofluidic technique, this method becomes much easier to perform, with less sample requirement and cross contamination.(7) Dynamic range is the ratio between the largest and smallest possible values of a changeable quantity. The dynamic range of measurement is limited by the saturation of a system or by physical limitations. The dynamic range of measurement is also limited by random noise or uncertainty that may be described as the defining the sensitivity of the system. In digital nucleic acid amplification system, the dynamic range can be increased by improving the number of compartmentalized reactors.(8) This is available by employing microfluidic method.(9) In this paper, we presented a digital nucleic acid amplification device based on microfluidic for absolute quantification and discussed the dynamic range and measurement uncertainty of it.

\section{MATERIAL AND METHODS}

\subsection{Fabrication}

The microfluidic device was fabricated by standard soft lithographic technique.(10) The devices were composed of two layers of the PDMS bonded to a glass coverslip. The chip patterns were designed using Corel DRAW X4. High-resolution transparent photomasks used for master producing were printed 
on transparent films. The master is produced by contact photolithography. The master consists of a positive relief of photo-resist on a silicon wafer and serves as a mold for PDMS. The mold was prepared by spin-coating photo-resist onto freshly cleaned and pre-dehydration silicon wafer. The thickness of the photo-resist is $80 \mu \mathrm{m}$. After the wafer was soft baked, the patterns were exposed by ultraviolent light with a mask aligner. PGMEA was used for nonexposed photo-resist dissolving. Then the wafer was cleaned with isopropyl alcohol. About 40 mins postbaking was necessary afterward. The final step of the mold producing process is baking to strengthen the structure for repeated usage.

The PDMS prepolymer mixture was prepared in a $5: 1$ ratio. The mixture needed $20 \mathrm{mins}$ degassing to remove the bubbles, and poured onto the mold afterward. Another blank PDMS was created on clean silicon wafer. Both wafers were baked at $80^{\circ} \mathrm{C}$ for 30mins. The PDMS was peeled off from the mold after solification, and sealed by glass coverslip with high-frequency plasma treatment. For the purpose of digital PCR performing, waterproof treatment is necessary because the temperature will up to $95^{\circ} \mathrm{C}$ during the thermal cycles. This challenge can be solved by inserting waterproof layer. The material of waterproof layer could be a piece of glass coverslip.

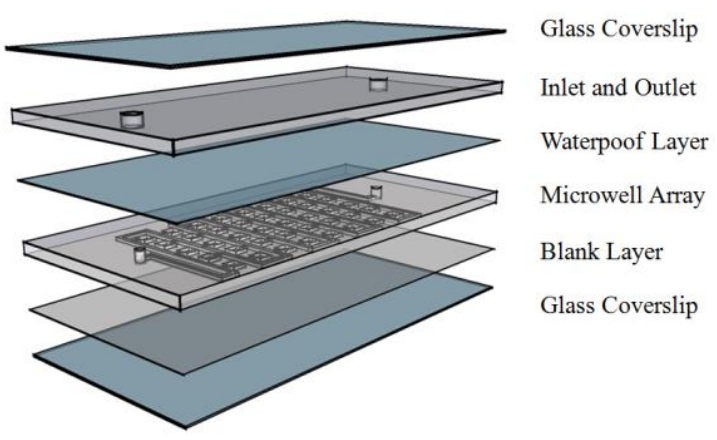

Figure 1. Schematic diagram of the microfluidic chip for dPCR

\subsection{Dynamic Range}

Dynamic range is a concept to measure and describe changeable quantity and is the ratio between the largest and smallest possible values of the quantity. The dynamic range of measurement is limited by the saturation of a system. Other physical limitations also may affect the range. In digital nucleic acid amplification system, the dynamic range is given by the concentration which the system becomes completely saturated (no chamber is negative), and can be increased by improving the number of compartmentalized reactors. Let $d$ be the number of empty chambers, thus:

$$
p(d)=\left(\left(W e^{-v / W}\right)^{d} \exp \left(W e^{-v / W}\right)\right) / d !
$$

$W$ is the total number of reaction chambers, $v$ is the total number of template molecules. The dynamic range of the digital nucleic acid amplification system is described above.

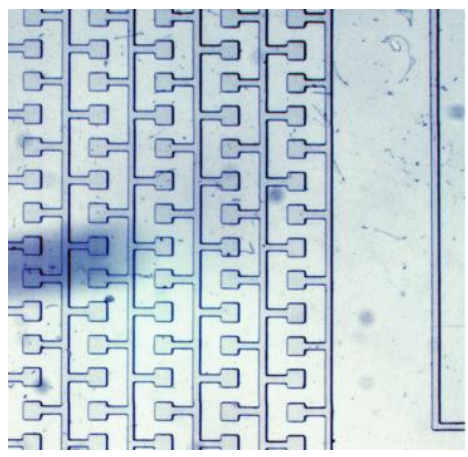

Figure 2. Microscope photograph of the structure in detail

\subsection{Measurement Uncertainty}

The relationship between the number of template molecules $B$, total number of chambers $D$, and the number of positive chambers $F$ is as follows:

$$
B=\log \left(1-\frac{F}{D}\right) / \log \left(1-\frac{1}{D}\right)
$$

$P_{v}$ is the chamber volume, target copy number concentration is $C_{p}$, therefore:

$$
\mathrm{C}_{\mathrm{p}}=\left(1 /\left(\mathrm{D} \times P_{v}\right)\right) \times\left(\log \left(1-\frac{F}{D}\right) / \log \left(1-\frac{1}{D}\right)\right)
$$

The possibility that a single chamber will be positive, $O$, has the standard deviation of:

$$
\sqrt{O(1-O) / \mathrm{D}}
$$

The true number of target molecules per chamber is $M$, therefore:

$$
M=-\ln (1-O)
$$

Thus:

$$
\partial M / \partial O=(-1 /(1-O)) \times(\partial(1-O) / \partial O)
$$

$V_{O}$, the standard uncertainty of $O$, is:

$V_{O}=\sqrt{O /(\mathrm{D}(1-O))}$

$V_{B}$, the standard uncertainty of $B$, therefore:

$V_{B}=\sqrt{D \times F /(D-F)}$

The combined relative uncertainty is:

$$
V_{C_{P}} / C_{P}=\sqrt{\left(V_{B} / B\right)^{2}+\left(V_{P_{V}} / P_{V}\right)^{2}}
$$

\section{CONCLUSION}

Nucleic acid quantification is essential in the field of medical and biological research, as well as variety of applications. The conventional method, qPCR is based on the calculation of the number of 
amplification cycles. The new method, dPCR, uses the same primers and probes as qPCR. However, it has higher sensitivity and precision, which is based on the concept 'divide and conquer'. This method is greatly promoted by the progress of nanofabrication and microfluidics. It is possible to produce hundreds, millions of nanoliter or even picoliter-scale chambers with these techniques. The more chambers, the greater the resolution, and is harder to be saturated. This means a higher dynamic range. In this paper, we have presented a microfluidic device for digital nucleic acid amplification and determination. The measurement precision and dynamic range of such system was clarified.

\section{REFERENCES}

[1] Mullis, K.B. \& Faloona, F.A. 1987. Specific synthesis of DNA in vitro via a polymerase-catalyzed chain reaction. Methods Enzymol. 155: 335-350.

[2] Mullis, K., Faloona, F., Scharf, S., Saiki, R., Horn, G., Erlich, H. 1986. Specific enzymatic amplification of DNA in vitro: the polymerase chain reaction. Cold Spring Harb Symp Quant Biol. 51 Pt 1: 263-273.

[3] Guo, M., Li, X., Zhang, S., Song, H., Zhang, W., Shang, X., Zheng, Y., Jiang, H., Lv, Q., Jiang, Y., Hao, H. 2015. Real-time quantitative RT-PCR detection of circulating tumor cells from breast cancer patients. Int J Oncol. 46(1): 281-289.

[4] Baker, M. 2012. Digital PCR hits its stride. Nature Methods. 9(6): 541-544.

[5] Vogelstein, B. \& Kinzler, K.W. 1999. Digital PCR. Proceedings Of the National Academy Of Sciences Of the United States Of America. 96(16): 9236-9241.

[6] Sanders, R., Mason, D.J., Foy, C.A., Huggett, J.F. 2013. Evaluation of digital PCR for absolute RNA quantification. PLoS One. 8(9): e75296.

[7] Ottesen, E.A., Hong, J.W., Quake, S.R., Leadbetter, J.R. 2006. Microfluidic digital PCR enables multigene analysis of individual environmental bacteria. Science. 314(5804): 1464-1467.

[8] Shen, F., Sun, B., Kreutz, J.E., Davydova, E.K., Du, W., Reddy, P.L., Joseph, L.J., Ismagilov, R.F. 2011. Multiplexed quantification of nucleic acids with large dynamic range using multivolume digital RT-PCR on a rotational SlipChip tested with HIV and hepatitis C viral load. J Am Chem Soc. 133(44): 17705-17712.

[9] Kreutz, J.E., Munson, T., Huynh, T., Shen, F., Du, W., Ismagilov, R.F. 2011. Theoretical design and analysis of multivolume digital assays with wide dynamic range validated experimentally with microfluidic digital PCR. Anal Chem. 83(21): 8158-8168.

[10] Kim, P., Lee, S.E., Jung, H.S., Lee, H.Y., Kawai, T., Suh, K.Y. 2006. Soft lithographic patterning of supported lipid bilayers onto a surface and inside microfluidic channels. Lab Chip. 6(1): 54-59. 